\title{
FIELDS OF PARALLEL VECTORS IN A RIEMANNIAN GEOMETRY*
}

BY

LUTHER PFAHLER EISENHART

1. Introduction. Consider a space $V_{n}$ of $n$ dimensions whose fundamental quadratic form is

$$
\varphi=g_{i j} d x^{i} d x^{j} \quad(i, j=1, \cdots, n),
$$

where the $g$ 's are subject only to the condition that their determinant

$$
g=\left|g_{i j}\right|
$$

is not zero.t We do not impose the condition that $\varphi$ is definite. We define the metric by

$$
d s^{2}=e g_{i j} d x^{i} d x^{j}
$$

where $e$ is plus or minus one according as $\varphi$ is positive or negative for a given set of values of the differentials.

Consider any curve $C$ in $V_{n}$ which is not of length zero, that is for which $\varphi$ is not zero, and assume that the coördinates $x^{i}$ at points of $C$ are functions of $s$ measured from some point of $C$ along it. Let $\lambda^{i}$ be the contravariant components of a unit vector field in $V_{n}$, that is

$$
g_{i j} \lambda^{i} \lambda^{j}=e,
$$

where $e$ is plus or minus one. In accordance with the concept of parallelism in $V_{n}$ due to Levi-Civita, we say that the vectors of the field at points of $C$ are parallel to one another with respect to $C$, if

$$
\frac{d x^{k}}{d s} \lambda^{i}, k \equiv \frac{d x^{k}}{d s}\left(\frac{\partial \lambda^{i}}{\partial x^{k}}+\left\{\begin{array}{c}
i \\
j k
\end{array}\right\} \lambda^{j}\right)=0,
$$

where $\lambda^{i}{ }_{k}$ is the covariant derivative of $\lambda^{i},\left\{\begin{array}{c}i \\ j k\end{array}\right\}$ being the Christoffel symbols of the second kind formed with respect to (1.1). When

* Presented to the Society, February 28, 1925.

† We use the customary convention of summation indicated by a repeated index. 563 


$$
\lambda_{, k}^{i}=0 \text {, }
$$

and only in this case, any two vectors are parallel to one another with respect to any curve passing through their points. In this case we have by definition a field of parallel vectors.

Fields of parallel vectors for geometries of paths have been considered by the author* and by Veblen and Thomas; the results of the latter can be used to give in invariantive form the conditions that a Riemannian space shall admit one or more fields of parallel vectors. In the present paper we derive the canonical forms of (1.1) for such spaces. $\neq$

2. Canonical coördinate system. Suppose that a $V_{n}$ is such that equations (1.5) admit $p$ linearly independent sets of solutions, $\lambda_{\sigma \mid}{ }^{i}$ for $\sigma=1, \cdots, p$ and $i=1, \cdots, n . \S$ In any other coördinate system, $x^{\prime i}$, the components $\lambda_{\sigma \mid}^{\prime}{ }^{i}$ are given by

$$
\lambda_{\sigma \mid}^{\prime}{ }^{i}=\lambda_{\sigma \mid}^{j} \frac{\partial x^{i}}{\partial x^{j}}
$$

If we take the system of $p$ differential equations

$$
X_{\sigma}(\theta) \equiv \lambda_{\sigma \mid}{ }^{j} \frac{\partial \theta}{\partial x^{j}}=0
$$

and apply the Poisson operator, we have in consequence of (1.5)

$$
\left(X_{\sigma} X_{\tau}-X_{\tau} X_{\sigma}\right) \theta \equiv 0
$$

Therefore equations (2.2) form a complete system, and consequently admit $n-p$ independent solutions. $\|$ If we take them for the coördinates $x^{\prime p+1}, \ldots, x^{\prime n}$, it follows from (2.1) that $\lambda_{\sigma \mid}^{\prime t}=0$ for $t=p+1, \cdots, n$. Again if we omit one of the equations from (2.2), say $X_{r} \theta=0$, the remaining system is complete and admits in addition to $x^{\prime p+1}, \ldots, x^{\prime n}$ another independent solution $x^{\prime r}$. In this way the $x^{\prime \prime}$ s are defined so that all of the components of the $\lambda$ 's are zero except those with the same

- Proceedings of the National Academy of Sciences, vol. 8 (1922), pp. 207-212. †These Transactions, vol. 25 (1923), p. 589.

¥ The case of spaces admitting one field of parallel rectors was considered by LeviCivita, Rendiconti del Circolo Matematico di Palermo, vol. 42 (1917), pp. 173-205.

$\S$ Unless otherwise stated, it will be assumed that Greek indices take the values $1, \cdots, p$ and Latin $1, \cdots, n$, throughout this paper.

|| Goursat, Leçons sur l'Intégration des Equations aux Dérivées Partielles du Premier Ordre, p. 52. 
subscript and superscript. If it is assumed that these vectors are unit vectors, we have in the new coördinate system

$$
\lambda_{\sigma \mid}{ }^{\sigma}=\frac{1}{\sqrt{e_{\sigma} g_{\sigma \sigma}}}, \quad \lambda_{\sigma \mid}{ }^{t}=0 \quad(\sigma=1, \cdots, p ; t=1, \cdots, n ; t \neq \sigma) .
$$

When these expressions are substituted in (1.5) we get

$$
\begin{aligned}
& \frac{\partial}{\partial x^{k}} \log V \overline{g_{\sigma \sigma}}-\left\{\begin{array}{c}
\sigma \\
k \sigma
\end{array}\right\}=0 \quad \text { ( } \begin{array}{l}
\sigma \\
\text { not summed), }
\end{array} \\
& \left\{\begin{array}{l}
j \\
\mid k \sigma
\end{array}\right\}=0 \quad(\sigma=1, \cdots, p ; j, l=1, \cdots, n ; j \neq \sigma) .
\end{aligned}
$$

If we multiply the first of these equations by $g_{\sigma l}$ and subtract the second multiplied by $g_{j l}$ and summed for $j$, we get the equivalent set of equations

that is

$$
g_{\sigma l} \frac{\partial}{\partial x^{k}} \log V \overline{g_{\sigma \sigma}}-[k \sigma, l]=0,
$$

$$
g_{\sigma l} \frac{\partial}{\partial x^{k}} \log g_{\sigma \sigma}-\frac{\partial g_{k l}}{\partial x^{\sigma}}-\frac{\partial g_{\sigma l}}{\partial x^{k}}+\frac{\partial g_{k \sigma}}{\partial x^{l}}=0 .
$$

For the case $k=\sigma$, these equations reduce to

$$
\frac{\partial}{\partial x^{\sigma}}\left(\frac{g_{\sigma l}}{\sqrt{e_{\sigma} g_{\sigma \sigma}}}\right)=e_{\sigma} \frac{\partial}{\partial x^{l}} \sqrt{e_{\sigma} g_{\sigma \sigma}} .
$$

In accordance with these equations we define $p$ functions $\psi_{\sigma}$ by

from which we have

$$
\sqrt{e_{\sigma} g_{\sigma \sigma}}=e_{\sigma} \frac{\partial \psi_{\sigma}}{\partial x^{\sigma}}, \quad \frac{g_{\sigma l}}{\sqrt{e_{\sigma} g_{\sigma \sigma}}}=\frac{\partial \psi_{\sigma}}{\partial x^{l}},
$$

$$
g_{\sigma l}=e_{\sigma} \frac{\partial \psi_{\sigma}}{\partial x^{\sigma}} \frac{\partial \psi_{\sigma}}{\partial x^{l}} .
$$

From these expressions it follows that $\psi_{\sigma}$ must involve $x^{\sigma}$, otherwise the space is of less than $n$ dimensions.

Again if neither $k$ nor $l$ in (2.4) is $\sigma$, we have

$$
g_{k l}=e_{\sigma} \frac{\partial \psi_{\sigma}}{\partial x^{k}} \frac{\partial \psi_{\sigma}}{\partial x^{l}}+\varphi_{k l \sigma}\left(\begin{array}{c}
\sigma=1, \cdots, p ; k, l=1, \cdots, n ; \\
k \neq \sigma, l \neq \sigma
\end{array}\right),
$$

where $\varphi_{k l \sigma}$ is a function independent of $x^{\sigma}$. 
From (2.5) and (2.6) it follows that for each value of $\sigma$ the fundamental form can be written

$$
\varphi=e_{\sigma}\left(d \psi_{\sigma}\right)^{2}+g_{r s} d x^{r} d x^{s} \quad(r, s=1, \cdots, n ; r \neq \sigma, s \neq \sigma),
$$

where $g_{r s}$ are independent of $x^{\sigma}$. If then we put $x^{\prime}{ }^{\sigma}=\psi^{\sigma}, x^{\prime j}=x^{j}(j \neq \sigma)$, the curves of parameter $x^{\prime \sigma}$ are the same as those of parameter $x^{\sigma}$, and these curves are geodesics.* Hence we have:

When $a V_{n}$ admits $p$ independent fields of parallel vectors, the vectors of each field are the tangent vectors to a congruence of geodesics.

Conversely if the fundamental form of any space is reducible to the form

$$
\varphi=e_{1} d x_{1}^{2}+g_{r s} d x^{r} d x^{s} \quad(r, s=2, \cdots, n),
$$

it is found from (2.4) that a necessary and sufficient condition that the tangents to the curves of parameter $x^{1}$ form a parallel field is that $g_{r s}$ be independent of $x^{1}$. In this case all the spaces $x^{1}=$ const. have the same fundamental form and consequently any one of them can be brought into coincidence with any other by a translation, that is by a motion in which each point describes the same distance along the geodesic normal to the subspace. In the case $p>1$ the space admits $p$ independent translations; thus any two of the sub-spaces of each of the family of sub-spaces $\psi_{\sigma}=$ const. can be brought into coincidence by a translation.

If in particular we take $\psi_{\sigma}=x^{\sigma}+\varphi_{\sigma}\left(x^{p+1}, \cdots, x^{n}\right)$ for $\sigma=1, \cdots, p$, it follows from (2.5) and (2.6) that for a $V_{n}$ with the fundamental form

$$
\varphi=e_{1}\left(d x^{1}\right)^{2}+\cdots+e_{p}\left(d x^{p}\right)^{2}+g_{\alpha \beta} d x^{\alpha} d x^{\beta} \quad(\alpha, \beta=p+1, \cdots, n)
$$

where $g_{\alpha \beta}$ are arbitrary functions of $x^{p+1}, \ldots, x^{n}$, the tangents to curves of parameters $x^{1}, \cdots, x^{p}$ form $p$ fields of parallel vectors. We proceed to the determination of the general solution.

3. When $p>1$. When $p>1$, we have from (2.5), on equating the two expressions for $g_{\sigma \tau}$,

$$
e_{\sigma} \frac{\partial \psi_{\sigma}}{\partial x^{\sigma}} \frac{\partial \psi_{\sigma}}{\partial x^{\tau}}=e_{\tau} \frac{\partial \psi_{\tau}}{\partial x^{\sigma}} \frac{\partial \psi_{\tau}}{\partial x^{\tau}}
$$

and from (2.5) and (2.6), on equating the two expressions for $g_{\tau \tau}$ and $g_{\sigma \sigma}$ respectively,

$$
\begin{aligned}
& e_{\sigma}\left(\frac{\partial \psi_{\sigma}}{\partial x^{\tau}}\right)^{2}+\varsigma_{\tau \tau \sigma}=e_{\tau}\left(\frac{\partial \psi_{\tau}^{\prime}}{\partial x^{\tau}}\right)^{2}, \\
& e_{\tau}\left(\frac{\partial \psi_{\tau}}{\partial x^{\sigma}}\right)^{2}+\varphi^{\prime \sigma \sigma \tau}=e_{\sigma}\left(\frac{\partial \psi_{\sigma}}{\partial x^{\sigma}}\right)^{2} \text {. }
\end{aligned}
$$

\footnotetext{
* Bianchi, Lezioni, vol. 1, p. 337.
} 
When these equations are differentiated with respect to $x^{\sigma}$ and $x^{\tau}$ respectively, we obtain

Hence either

$$
\begin{aligned}
& e_{\sigma} \frac{\partial \psi_{\sigma}}{\partial x^{\tau}} \frac{\partial^{2} \psi_{\sigma}}{\partial x^{\sigma} \partial x^{\tau}}-e_{\tau} \frac{\partial \psi_{\tau}}{\partial x^{\tau}} \frac{\partial^{2} \psi_{\tau}}{\partial x^{\sigma} \partial x^{\tau}}=0 \\
& e_{\sigma} \frac{\partial \psi_{\sigma}^{\prime}}{\partial x^{\sigma}} \frac{\partial^{2} \psi_{\sigma}^{\prime}}{\partial x^{\sigma} \partial x^{\tau}}-e_{\tau} \frac{\partial \psi_{\tau}}{\partial x^{\sigma}}-\frac{\partial^{2} \psi_{\tau}}{\partial x^{\sigma} \partial x^{\tau}}=0 .
\end{aligned}
$$

$$
\frac{\partial^{2} \psi_{\sigma}}{\partial x^{\sigma} \partial x^{\tau}}=\frac{\partial^{2} \psi_{\tau}^{\prime}}{\partial x^{\sigma} \partial x^{\tau}}=0
$$

or

$$
\frac{\partial \psi_{\sigma}}{\partial x^{\tau}} \frac{\partial \psi_{\tau}}{\partial x^{\sigma}}=\frac{\partial \psi_{\sigma}}{\partial x^{\sigma}} \frac{\partial \psi_{\tau}}{\partial x^{\tau}}
$$

If (3.4) holds, it follows from (3.1) and (3.2) that $\varphi_{\sigma \sigma \tau}=\varphi_{\tau \tau \sigma}=0$. This case will be considered in $\S 7$.

From equations of the form (3.3) it follows that the functions $\psi_{\sigma}$ must be of the form

$$
\begin{aligned}
\psi_{\sigma}= & e_{\sigma} f_{\sigma}\left(x^{\sigma}, x^{p+1}, \cdots, x^{n}\right) \\
& +F_{\sigma}\left(x^{1}, \cdots, x^{\sigma-1}, x^{\sigma+1}, \cdots, x^{p}, x^{p+1}, \cdots, x^{n}\right) \quad(\sigma=1, \cdots, p) .
\end{aligned}
$$

From the remark following (2.5) it is seen that $f_{\sigma}$ must involve $x^{\sigma}$. Moreover it is understood that $f_{\sigma}$ does not have any additional function independent of $x^{\sigma}$, since all such terms are included in $F_{\sigma}$.

If $\partial \psi_{\sigma} / \partial x^{\tau}=0$, so also is $\partial \psi_{\tau} / \partial x^{\sigma}=0$, as follows from (3.1). These exceptional cases are taken care of by (3.5).

Substituting from (3.5) in (3.1), we get

$$
\frac{\frac{\partial F_{\tau}}{\partial x^{\sigma}}}{\frac{\partial f_{\sigma}}{\partial x^{\sigma}}}=\frac{\frac{\partial F_{\sigma}}{\partial x^{\tau}}}{\frac{\partial f_{\tau}}{\partial x^{\tau}}} .
$$

Since the first and second members of this equation are independent of $x^{\tau}$ and $x^{\sigma}$ respectively, they are independent of both. Consequently we replace (3.6) by

$$
\frac{\partial F_{\tau}}{\partial x^{\sigma}}=\frac{\partial f_{\sigma}}{\partial x^{\sigma}} \omega_{\tau \sigma}, \quad \frac{\partial F_{\sigma}}{\partial x^{\tau}}=\frac{\partial f_{\tau}}{\partial x^{\tau}} \omega_{\sigma \tau} \quad(\sigma, \tau=1, \cdots, p ; \sigma \neq \tau),
$$

where $\omega_{\tau \sigma}\left(=\omega_{\sigma \tau}\right)$ is independent of $x^{\sigma}$ and $x^{\tau}$. 
4. When $p=2$. When $p=2$, we have from (3.5) and (3.7), since $\omega_{\sigma \tau}$ is symmetric,

$$
\psi_{\sigma}^{\prime}=e_{\sigma} f_{\sigma}+A f_{\tau}+B_{\sigma} \quad(\sigma, \tau=1,2 ; \sigma \neq \tau),
$$

where $A, B_{1}$ and $B_{2}$ are independent of $x^{1}$ and $x^{2}$.

For the general case we have from (2.5) and the expressions for $g_{\sigma l}$ from (2.6) the equations of condition

$$
e_{\sigma} \frac{\partial \psi_{\sigma}^{\prime}}{\partial x^{\sigma}} \frac{\partial \psi_{\sigma}}{\partial x^{l}}=e_{\tau} \frac{\partial \psi_{\tau}}{\partial x^{\sigma}} \frac{\partial \psi_{\tau}}{\partial x^{l}}+\varphi_{\sigma \tau}\left(\begin{array}{l}
\sigma=1, \cdots, p ; l=1, \cdots, n ; \\
l, \sigma, r \neq
\end{array}\right) .
$$

When $p=2$ and we take $\sigma=1, \tau=2, l=3, \cdots, n$, this condition for the expressions (4.1) becomes

$$
\frac{\partial f_{1}}{\partial x^{1}}\left[f_{2} \frac{\partial A}{\partial x^{l}}+\frac{\partial}{\partial x^{l}}\left(e_{1} f_{1}+B_{1}\right)-e_{2} A_{2} \frac{\partial}{\partial x^{l}}\left(A f_{1}+B_{2}\right)\right]-\varphi_{1 \sigma 2}=0 .
$$

Since $\partial f_{1} / \partial x^{1} \neq 0$ and all the terms except $f_{2}$ are independent of $x^{2}$, it follows that $A$ must be a constant.

There remain for consideration the equations which arise from (2.6) on equating the expressions for each $g_{k l}(k, l=3, \cdots, n)$ for $\sigma=1,2$. Since $\varphi_{k l 1}$ and $\varphi_{k l 2}$ may be any functions not involving $x^{1}$ and $x^{2}$ respectively, we find that these conditions are satisfied, because $A$ is a constant. In arriving at this result we take

$$
\begin{aligned}
& \varphi_{k l 1}=e_{2} \frac{\partial}{\partial x^{k}}\left(e_{2} f_{2}+B_{2}\right) \frac{\partial}{\partial x^{l}}\left(e_{2} f_{2}+B_{2}\right)-e_{1} \frac{\partial}{\partial x^{k}}\left(A f_{2}+B_{1}\right) \frac{\partial}{\partial x^{l}}\left(A f_{2}+B_{1}\right) \\
& +e_{1} \frac{\partial B_{1}}{\partial x^{k}} \frac{\partial B_{1}}{\partial x^{l}}+C_{k l}
\end{aligned}
$$

where $C_{k l}$ are arbitrary functions of $x^{3}, \ldots, x^{n}$. Then we have

$$
\begin{aligned}
g_{k l}=\sum_{\sigma}^{1,2} e_{\sigma} \frac{\partial}{\partial x^{k}}\left(e_{\sigma} f_{\sigma}+B_{\sigma}\right) \frac{\partial}{\partial x^{l}}\left(e_{\sigma} f_{\sigma}+B_{\sigma}\right) & \\
& +A\left(\frac{\partial f_{1}}{\partial x^{k}} \frac{\partial f_{2}}{\partial x^{l}}+\frac{\partial f_{1}}{\partial x^{l}} \frac{\partial f_{\mathfrak{z}}}{\partial x^{k}}\right)+C_{k l} .
\end{aligned}
$$

Hence we have the following theorem:

When $f_{1}$ and $f_{2}$ are arbitrary functions of $x^{1}, x^{3}, \cdots, x^{n}$ and $x^{2}, x^{3}, \cdots, x^{n}$ respectively, $A$ is an arbitrary constant, and $B_{1}$ and $B_{2}$ are arbitrary junc- 
tions of $x^{3}, \ldots, x^{n}$, the functions (4.1) determine a $V_{n}$ with two fields of parallel vectors; and $C_{k c l}$ in (4.4) are also arbitrary functions of $x^{3}, \ldots, x^{n}$.

5. When $p=3$. If $p>2$ and we express the condition of integrability of two of the first set of equations (3.7) for two values of $\sigma$ different from $\pi$, say $\alpha$ and $\beta$, we find by considerations similar to those applied to (3.6)

$$
\frac{\partial \omega_{\tau \alpha}}{\partial x^{\beta}}=\frac{\partial f_{\beta}^{\prime}}{\partial x^{\beta}{ }^{\omega} \tau \alpha \beta}, \quad \frac{\partial \omega \tau \beta}{\partial x^{\alpha}}=\frac{\partial f_{\alpha}}{\partial x^{\alpha}} \omega_{\tau \beta \alpha} \quad\left(\begin{array}{l}
\alpha, \beta, \tau=1, \cdots, p ; \\
\alpha, \beta, \tau \neq
\end{array}\right),
$$

where $\omega_{\tau \alpha \beta}\left(=\omega_{\tau \beta \alpha}\right)$ is independent of $x^{\tau}, x^{\alpha}$ and $x^{\beta}$, and consequently

$$
\omega_{\tau \alpha}=f_{\beta} \omega_{\tau \alpha \beta}+Q_{\tau \alpha \beta},
$$

where $\varrho_{\tau \alpha \beta}$ is independent of $x^{\tau}, x^{\alpha}$ and $x^{\beta}$. Since $\omega_{\tau \alpha}=\omega_{\alpha \tau}, \omega_{\tau \alpha \beta}$ must be symmetric in $\tau$, $\alpha$ and $\beta$, and $\varrho_{\tau \alpha \beta}$ in $\tau$ and $\alpha$. For $p=3$, we have from (3.7) and (5.1)

$$
F_{\sigma}=A \prod_{\alpha}^{\prime}\left(f_{\alpha}\right)+\sum_{\sigma}^{\prime} B_{\sigma \alpha} f_{\alpha}+C_{\sigma} \quad(\sigma=1,2,3)
$$

where $\prod_{\alpha}^{\prime} l_{\sigma}^{\prime}\left(f_{\alpha}\right)$ means the product of all the $f^{\prime \prime}$ s except $f_{\sigma}$ and $\sum_{\alpha}^{\prime}$ indicates the sum for $\alpha$ except $\alpha=\sigma$; also $A, B_{\sigma \alpha}=B_{\alpha \sigma}$ and $C_{\sigma}$ are functions independent of $x^{1}, x^{2}$ and $x^{3}$.

If we substitute in (4.2) the expressions $\psi_{\sigma}=e_{\sigma} f_{\sigma}+F_{\sigma}$, where $F_{\sigma}$ is given by (5.2) and similarly for $\psi_{\tau}$, we obtain equations of the form

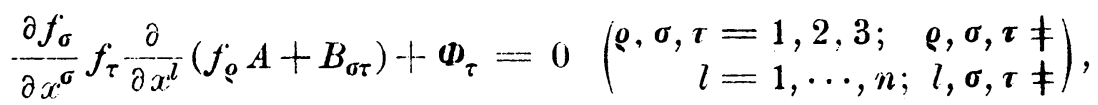

where $\Phi_{\tau}$ does not involve $x^{\tau}$. Hence

$$
\frac{\partial}{\partial x^{l}}\left(f_{\underline{Q}} A+B_{\sigma \tau}\right)=0
$$

For $l=\varrho$ we get $A=0$, and for other values of $l$ it follows that the $B$ 's are constant.

When as in $\$ 4$ we equate the three values of each $g_{k l}(k, l=4, \cdots, n)$ obtained from (2.6) by taking $\sigma=1,2,3$, we find that these equations are consistent in view of the above results. Moreover, the functions $\varphi_{k l v}$ are of the forms 


$$
\begin{aligned}
\varphi_{k l \sigma}=e_{\tau} \frac{\partial}{\partial x^{k}}\left(e_{\tau} f_{\tau}+B_{\tau \varrho} f_{\underline{\varphi}}+C_{\tau}^{\prime}\right) \frac{\partial}{\partial x^{l}}\left(e_{\tau} f_{\tau}\right. & \left.+B_{\tau \varrho} f_{\underline{\varphi}}+C_{\tau}\right) \\
& -e_{\alpha} B_{\sigma \tau}^{2} \frac{\partial f_{\tau}}{\partial x^{k}}-\frac{\partial f_{\tau}}{\partial x^{l}}+D_{k l \sigma \tau}
\end{aligned}
$$

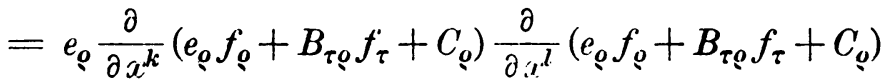

$$
\begin{aligned}
& -e_{\alpha} B_{\sigma o}^{2} \frac{\partial f_{\rho}}{\partial x^{k}} \frac{\partial f_{o}}{\partial x^{l}}+D_{k l \sigma \varphi} \\
& (\varrho, \sigma, x=1,2,3 ; \quad \varrho, \sigma, \tau \neq) \text {, }
\end{aligned}
$$

where $D_{k l \sigma \tau}\left(=D_{k l \tau \sigma}\right)$ is independent of $x^{\sigma}$ and $x^{\tau}$. These two expressions for $\varphi_{k l \sigma}$ are seen to be consistent, if we take

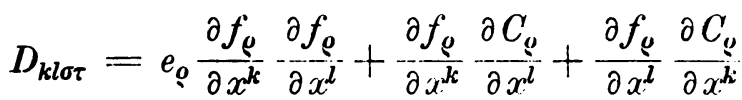

$$
\begin{aligned}
& -e_{\tau} \frac{\partial}{\partial x^{k}}\left(B_{\tau \varrho} f_{\underline{\rho}}+C_{\tau}\right) \frac{\partial}{\partial x^{l}}\left(B_{\tau \rho} f_{\varrho}+C_{\tau}\right) \\
& -e_{\sigma} \frac{\partial}{\partial x^{k}}\left(B_{\sigma \underline{\varphi}} f_{\underline{\rho}}+C_{\sigma}\right) \frac{\partial}{\partial x^{l}}\left(B_{\sigma \underline{\varphi}} f_{\underline{\rho}}+C_{\sigma}\right)+E_{k l \sigma \tau \underline{\rho}} \text {, }
\end{aligned}
$$

where $E_{\text {kloto }}$ are arbitrary functions of $x^{4}, \cdots, x^{\alpha}$. Hence we have the following theorem:

The functions $\psi_{\sigma}$ defined by

$$
\psi_{\sigma}=-e_{\sigma} f_{\sigma}+\sum_{\omega}^{\prime} B_{\sigma \alpha} f_{\alpha}+C_{\sigma} .
$$

where the $f_{0}^{\prime}$ are arbiticuly functions of $x^{6}, x^{4}, \cdots, x^{n}$, the $B^{\prime} s$ are constants symmetric in their indices and the $C^{\prime}$ 's are arbitrary finctions of $x^{4}, \ldots, x^{n}$, determine a $V_{n}$ with three fields of parallel rectors; and $E_{k l o t o}$ in (5.4) are arbitrary finctions of $x^{4}, \cdots, x^{n}$.

6. When $p>3$. In this case we have from (5.1) equations of the form

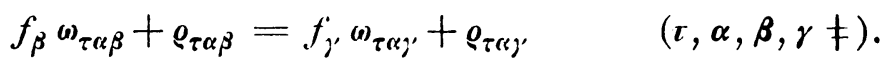

If this equation be differentiated with respect to $x^{\beta}$ and $x^{\gamma}$, the resulting equation leads, by considerations similar to those applied to (3.6), to

$$
\frac{\partial \omega \omega_{\tau \alpha \beta}}{\partial x^{\gamma}}=\frac{\partial f_{\gamma}}{\left.\partial x^{\gamma}{ }^{\prime}\right)_{\tau(\alpha \beta \gamma}, .} \quad \frac{\partial \omega_{\tau \alpha \gamma}}{\partial x^{\beta}}=\frac{\partial f_{\beta}}{\partial x^{\beta}} \omega_{\tau(\alpha, \beta},
$$


where $\omega_{\tau \alpha \beta \gamma}$ is symmetric in $\beta$ and $\gamma$ and is independent of $x^{\tau}, x^{\alpha}, x^{\beta}$ and $x^{\gamma}$. From these equations we have

and from (6.1)

$$
\omega_{\tau \alpha \beta}=f_{\gamma} \omega_{\tau \alpha \beta \gamma}+\varrho_{\tau \kappa \beta \gamma \gamma}, \quad \omega_{\tau \alpha \gamma}=f_{\beta} \omega_{\tau \alpha \gamma \beta}+\varrho_{\tau \alpha \gamma \beta},
$$

$$
\varrho_{\tau \alpha \beta}=\varrho_{\tau \alpha \gamma \beta} f_{\gamma}+\bar{\varrho}_{\tau \alpha \beta \gamma}, \quad \varrho_{\tau \alpha \gamma}=\varrho_{\tau \alpha \beta \gamma} f_{\beta}+\bar{\varrho}_{\tau \alpha \gamma \beta} .
$$

By continuing this process, we find as the general solution of (3.7)

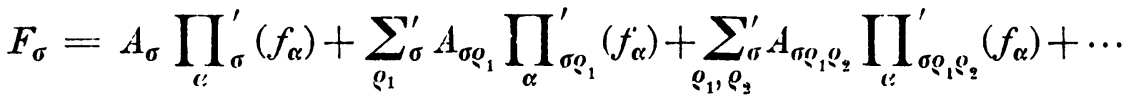

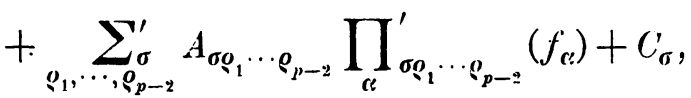

where the $A^{\prime}$ 's and $C^{\prime}$ 's are functions of $x^{p+1}, \cdots, x^{n}$, the symbol $\sum_{\varrho_{1} \cdots \varrho_{r}}^{\prime}$ means the sum for all different combinations of different $\rho$ 's taking the values $1, \cdots, p$ except $\sigma$ and only one such term for each combination; and

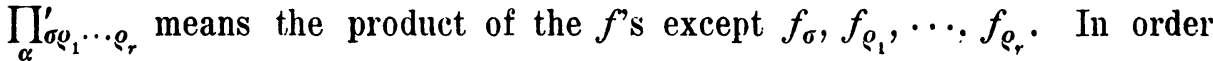
that (3.1) be satisfied, we must have $A_{1}=A_{2}=\ldots=A_{p} \equiv A$ and the other $A$ 's must satisfy the conditions

$$
A_{\sigma \varrho_{1} \cdots \varrho_{r}}=A_{\tau \varrho_{1} \cdots \varrho_{r}} \quad\left(\sigma, \tau, \varrho_{1}, \cdots, \varrho_{r} \neq\right) .
$$

When these expressions are substituted in (4.2), the latter are reducible to the form $\boldsymbol{\Phi}_{\sigma} f_{\tau}+\boldsymbol{\Psi}_{\sigma}=\mathbf{0}$ where $\boldsymbol{\Phi}_{\sigma}$ and $\boldsymbol{\Psi}_{\sigma}$ are independent of $\boldsymbol{x}^{\tau}$. Accordingly they must vanish. The first of these conditions is

where

$$
\begin{aligned}
& \frac{\partial}{\partial x^{l}}\left[A \prod_{\sigma \tau}^{\prime}\left(f_{c}^{\prime}\right)+\sum_{\varrho_{1}}^{\prime} A_{\sigma \varrho_{1}} \prod_{\sigma \tau \varrho_{1}}^{\prime}\left(f_{c l}^{\prime}\right)\right. \\
& \left.\quad+\sum_{\varrho_{1}, \varrho_{2}}^{\prime} A_{\sigma \varrho_{1} \rho_{2}} \prod_{\sigma \tau \varrho_{1} o_{2}}^{\prime}\left(f_{c}^{\prime}\right)+\cdots+D_{\sigma \tau}\right]=0 \quad(l, \sigma, \tau \neq),
\end{aligned}
$$

$$
D_{\sigma \tau}=A_{\sigma \varphi_{1} \cdots g_{p-2}}=A_{\tau \varrho_{1} \cdots o_{p-2}} \quad\left(\varrho_{1}, \cdots, \varrho_{p-2}, \sigma, \tau \neq\right) .
$$

When $l$ takes a value $1, \cdots, p$, different from $\sigma$ and $\tau$, say $\varrho$, the above equation becomes

$$
\begin{aligned}
& \frac{\partial f_{\rho}}{\partial x^{\rho}}\left[A \prod_{\rho \sigma \tau}^{\prime}\left(f_{\alpha}\right)+\sum_{\varrho_{1}}^{\prime}{ }_{\rho \sigma \tau} A_{\sigma \rho_{1}} \prod_{\rho \sigma \tau \rho_{1}}^{\prime}\left(f_{\alpha}\right)\right.
\end{aligned}
$$

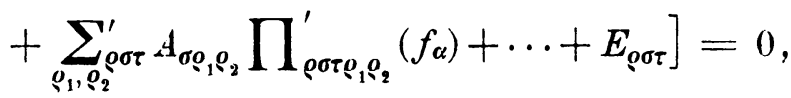


where

$$
E_{\varrho \sigma \tau}=A_{\sigma \varrho_{1} \cdots \varrho_{p-8}}=A_{\tau \varrho_{1} \cdots \varrho_{p-8}}=A_{\varrho \varrho_{1} \cdots \varrho_{p-8}}\left(\varrho_{1}, \cdots, \varrho_{p-3}, \varrho, \sigma, \tau \neq\right) .
$$

Since $\partial f_{\varrho} / \partial x^{\rho} \neq 0$, it follows from the above equations that $A$ and all the $A$ 's with less than $p-1$ indices are zero. And from (6.4) for $l=p+1, \ldots, n$ we have that the $D$ 's (6.5) are constants. Hence from (3.5) and (6.2) we have

$$
\psi_{\sigma}=e_{\sigma} f_{\sigma}+\sum_{\tau}^{\prime} D_{\sigma \tau} f_{\tau}+C_{\sigma}(\sigma, \boldsymbol{r}=1, \cdots, p ; \sigma \neq \boldsymbol{\sigma}),
$$

where the $D$ 's are constants symmetric in the subscripts and $C_{\sigma}$ are arbitrary functions of $x^{p+1}, \cdots, x^{n}$.

When now we equate the $p$ expressions for each of the functions $g_{k l}(k, l=p+1, \cdots, n)$ obtained from (2.6) by taking $\sigma=1, \cdots, p$, we find that these equations are consistent and that the functions $\varphi_{k l \sigma}$ involve additive arbitrary functions of $x^{p+1}, \cdots, x^{n}$ as shown in $\S \S 4,5$. Thus the theorems of these sections can be generalized to any value of $p<n$.

7. When one or more of the functions $\varphi_{\sigma \sigma \tau}$ in (3.2) are zero. If $\varphi_{\sigma \sigma \tau}=0$ in the second of (3.2), it follows from this equation that $e_{\sigma}=e_{\tau}$, if the functions are to be real. From this equation and (3.1) we have then

$$
\left(\frac{\partial \psi_{\tau} \tau}{\partial x^{\sigma}}\right)^{2}=\left(\frac{\partial \psi_{\sigma}}{\partial x^{\sigma}}\right)^{2}, \quad\left(\frac{\partial \psi_{\tau}}{\partial x^{\tau}}\right)^{2}=\left(\frac{\partial \psi_{\sigma}}{\partial x^{\tau}}\right)^{2},
$$

and from the first of (3.2) we find that $\varphi_{\tau \tau \sigma}==0$.

If we take $\sigma=1, \tau=2$, from the first of (7.1) it follows that

$$
\psi_{2}=\varepsilon \psi_{1}+\varphi_{1},
$$

where $\varepsilon^{2}=1$ and $\varphi_{1}$ is independent of $x^{1}$. Substituting in the second of (7.1), we obtain

$$
\left(2 \varepsilon \frac{\partial \psi_{1}}{\partial x^{2}}+\frac{\partial \varphi_{1}}{\partial x^{8}}\right) \frac{\partial \varphi_{1}}{\partial x^{2}}=0 .
$$

Assume that $\partial \varphi_{1} / \partial x^{2} \neq 0$; then

$$
\psi_{1}=\frac{1}{2 \epsilon}\left(\varphi_{2}-\varphi_{1}\right)
$$

where $\varphi_{2}$ is independent of $x^{2}$, and from (7.2)

$$
\psi_{2}=\frac{1}{2}\left(\varphi_{2}+\varphi_{1}\right) .
$$


Substituting in (3.1), we get $\partial \varphi_{2} / \partial x^{1}=0$. In this case $\psi_{1}$ is independent of $x^{1}$ and from (2.5) we get $g_{1 l}=0(l=1, \ldots, n)$, which is impossible. Hence $\varphi_{1}$ must be independent of $x^{2}$, and accordingly we write (7.2) in the form

$$
\psi_{z}=\varepsilon \psi_{1}+\varphi_{12},
$$

where $\varphi_{12}$ is independent of $x^{1}$ and $x^{2}$.

When the expression (7.4) is substituted in (4.2) for $\sigma=1, v=2$, we get

$$
\varepsilon \frac{\partial \psi_{1}}{\partial x^{1}} \frac{\partial \varphi_{12}}{\partial x^{l}}+\varphi_{1 l 2}=0 \quad(l=3, \cdots, n) .
$$

Differentiating with respect to $x^{2}$, we obtain

Hence either

$$
\frac{\partial^{2} \psi_{1}}{\partial x^{1} \partial x^{8}} \frac{\partial \varphi_{12}}{\partial x^{l}}=0
$$

or $\varphi_{12}$ is a constant. In the latter case we have from (2.5) and (7.4)

$$
g_{1 l}=e_{1} \frac{\partial \psi_{1}}{\partial x^{l}} \frac{\partial \psi_{1}}{\partial x^{l}}, \quad g_{2 l}=e_{1} \frac{\partial \psi_{1}}{\partial x^{2}} \frac{\partial \psi_{1}}{\partial x^{l}} \quad(l=1, \ldots, n) .
$$

Then the fundamental form may be written

$$
\varphi=e_{1}\left(d \psi^{1}\right)^{2}+g_{\alpha \beta} d x^{\alpha} d x^{\beta} \quad(\alpha, \beta=3, \cdots, n),
$$

that is, the space is a $V_{n-1}$ at most. Hence (7.5) is the only solution.

It is readily found that the two expressions for any $g_{k l}(k, l,=3, \ldots, n)$ and for $\sigma=1,2$ from (2.6) are consistent under the above conditions.

From the foregoing considerations it follows that the particular cases when one or more of the functions $\varphi_{\sigma \sigma \tau}$ in (3.2) are zero arise only when constants $D_{\sigma \tau}$ in (6.6) have certain values.

Princeton University,

Princeton, N. J. 